Revista Destaques Acadêmicos, Lajeado, v. 10, n. 3, 2018. ISSN 2176-3070

DOI: http://dx.doi.org/10.22410/issn.2176-3070.v10i3a2018.1968

http://www.univates.br/revistas

\title{
DESAFIOS E DESCOBERTAS SOBRE O ATENDIMENTO INTERDISCIPLINAR EM PACIENTES ONCOLÓGICOS: UMA REVISÃO INTEGRATIVA
}

\author{
Caroline Schonhalz Veloso ${ }^{1}$, Daniel Silveira da Silva ${ }^{2}$
}

\begin{abstract}
Resumo: O presente estudo buscou analisar através da revisão integrativa da literatura, as pesquisas produzidas na oncologia com tema de interdisciplinaridade. $\mathrm{O}$ levantamento bibliográfico abrangeu as publicações nacionais nos últimos 6 anos, sendo identificados 18 artigos que compuseram a amostra. Observou-se que na literatura atual houve uma diminuição das publicações relacionadas ao trabalho interdisciplinar na segunda metade do período estudado. Foi possível demonstrar a ampla variedade de profissionais que são importantes para o tratamento do paciente para que o mesmo seja atendido de forma integral como é preconizado. O tema é complexo e exige mais reflexões e discussões, envolve demandas públicas e produção de normas e diretrizes para a execução dessa forma de trabalho. Sugere-se, portanto, novos estudos para aprofundar a temática e difundir esta forma de assistência.
\end{abstract}

Palavras-chave: interdisciplinar, oncologia, equipe e neoplasia

\section{INTRODUÇÃO}

Os avanços diagnósticos e terapêuticos têm favorecido a sobrevivência das pessoas com doenças graves, com a redução da mortalidade. Por outro lado, as recentes estimativas apontam para o aumento de internações hospitalares de pacientes com doenças oncológicas nos próximos anos (INCA; 2002). Dentre outros motivos, esse é um, pelo qual devemos aprimorar o atendimento ao paciente oncológico em ambiente hospitalar, independentemente do estágio da doença, agravantes e prognóstico.

1 Pós-graduanda em Atenção Multidisciplinar em Oncologia. Centro Universitário UNIVATES. Enfermeira no Hospital Bruno Born.

2 Mestre em Ambiente e Desenvolvimento, Especialista e docente no curso Atenção Multidisciplinar em Oncologia. Centro Universitário UNIVATES. Enfermeiro no Centro Regional de Oncologia - CRON. 
Dessa forma, se faz necessário uma equipe multiprofissional atuante, na qual os diferentes integrantes colaboram para o desenvolvimento das atividades junto ao paciente de forma integral. Este último termo, relacionado com a integralidade da atenção, é entendido como a ação global que tem sido frequentemente associada ao tratamento com dignidade, respeito, qualidade, acolhimento e vínculo, compreendendo o ser humano como biopsicosocial e espiritual (GONZÁLEZ; ALMEIDA, 2010).

A Organização Mundial da Saúde (OMS) estima uma incidência de câncer de aproximadamente 27 milhões de casos para o ano 2030. Neste mesmo período, estima-se que ocorram 17 milhões de mortes por câncer e haja 75 milhões de pessoas vivas convivendo com a doença. Atualmente, mais de $70 \%$ dos óbitos por câncer ocorrem em países em desenvolvimento, nos quais os recursos disponíveis para prevenção, diagnóstico e tratamento da doença, são limitados ou inexistentes. Os grupos desfavorecidos estão geralmente mais expostos a fatores de risco de câncer, como tabagismo, alcoolismo e agentes infecciosos, além de possuírem menos acesso aos serviços de saúde (WHO, 2002).

As neoplasias são responsáveis por cerca $13 \%$ de todas as mortes no mundo, e a maioria dos indivíduos com câncer apresenta doença avançada e incurável no momento do diagnóstico. Além de provocar transtornos aos indivíduos, através da perda de equilíbrio físico, emocional, social e espiritual, o câncer também onera o Estado, com elevados custos no processo de diagnóstico, tratamento e controle de sintomas durante a evolução da doença, além daqueles decorrentes da perda ou redução da capacidade produtiva (WHO, 2002).

Para atender o ser humano em sua integralidade, ou seja, compreendendo-o como um ser composto pelas dimensões física, emocional, social e espiritual, fazem-se necessárias ações de uma equipe interdisciplinar, que exige a aquisição de novas capacidades técnicas e pedagógicas tanto por parte dos gestores quanto dos trabalhadores. É um processo de aprendizado coletivo, cuja possibilidade de sucesso está fundamentada no grande potencial resolutivo e de satisfação que ela pode trazer aos usuários e trabalhadores. É importante para a humanização, porque os serviços e os saberes profissionais muitas vezes segmentam os sujeitos em partes ou patologias. Essas equipes são uma forma de resgatar o compromisso com a pessoa, reconhecendo toda a complexidade do seu adoecer e do seu projeto terapêutico (BRASIL, 2007).

O presente estudo teve por objetivo caracterizar a produção científica brasileira sobre atendimento interdisciplinar em pacientes oncológicos. Para tanto, buscou-se identificar os aspectos relacionados à esse tipo de atendimento, bem como identificar na literatura qual a relevância desse atendimento para o tratamento da doença oncológica. 


\section{PROCEDIMENTOS METODOLÓGICOS}

Optou-se por realizar uma revisão integrativa para alcançar os objetivos propostos. Esse método tem a finalidade de reunir e sintetizar resultados de pesquisas sobre um delimitado tema ou questão, de maneira sistemática e ordenada, contribuindo para o aprofundamento do conhecimento do tema investigado. Desde 1980 a revisão integrativa é relatada na literatura como método de pesquisa (ROMAN, 1998). Seguiu-se os seis passos metodológicos propostos por Ganong: 1. Selecionar as hipóteses ou questões para a revisão; 2. Estabelecer os critérios para a seleção da amostra; 3 . Apresentar as características da pesquisa primária; 4. Análise dos dados; 5 . Interpretação dos resultados; 6. Apresentação da revisão ( GANONG 1987).

A coleta dos dados foi realizada em janeiro de 2017 por meio da busca eletrônica (Internet) nas seguintes bases: Literatura Latino-americana e do Caribe em Ciências da Saúde (LILACS) e na Scientific Electronic Library Online (SciELO). Utilizou-se os seguintes descritores padronizados no Descritores em Ciências da Saúde (DeCS): "interdisciplinar", "oncologia", equipe " e "neoplasia". Sendo que o agrupamento deu-se da seguinte forma: interdisciplinar and oncologia e equipe and neoplasia.

Os critérios utilizados para a seleção da amostra foram: artigos indexados, publicados em periódicos nacionais, no período compreendido entre 2011 e 2016, disponíveis na íntegra, em português, que abordassem a temática do câncer e interdisciplinaridade. Os critérios de exclusão foram: artigos pagos, dissertações, teses e resumos.

A questão norteadora deste estudo foi: Que achados a produção científica brasileira traz sobre atendimento interdisciplinar em pacientes oncológicos?

A busca às bases de dados inicial resultou em 23 artigos acessados com utilização dos descritores controlados, destes, 5 não abordavam a temática da pesquisa. Resultando em 18 artigos.

\section{RESULTADOS E DISCUSSÃO}

Dos 18 artigos selecionados, 7 (38,8\%) deles estavam disponíveis na base de dados SciELO, enquanto que $11(61,1 \%)$ na LILACS. É possível inferir que o maior número de publicações encontradas na LILACS deve-se ao fato desta base existir há 31 anos, portanto tem um número maior de artigos, enquanto o SciELO tem 18 anos de acesso aberto.

Ao analisar a quantidade de publicações por ano, em 2011 encontrouse 5 artigos correspondendo a 27,7\% da amostra. Em 2012 foram 3 artigos $(16,6 \%)$, seguidos de 6 artigos (33,3\%) em 2013 e 2 artigos (11,1\%) em 2014, enquanto que em 2015 e 2016 somente 1 artigo em cada ano (5,5\%). Sendo assim, relacionando o tema e as publicações referentes aos períodos do estudo, pode-se dizer que houve uma diminuição das pesquisas relacionadas ao tema. 
Os dados referentes aos artigos que compuseram a amostra estão apresentados na Tabela 1.

Em relação ao delineamento da pesquisa, as apresentações foram diversificadas. Em muitos artigos foram utilizados duas ou mais formas de metodologia. Com predomínio das pesquisas Descritivas, Exploratórias e Qualitativa.

\section{Análise dos artigos na íntegra e respectivos aspectos relevantes:}

Tabela 1: Identificação dos artigos conforme título, ano, método, aspectos relevantes, autores e base de dados.

\begin{tabular}{|c|c|c|c|c|c|c|}
\hline $\mathrm{N}^{\mathrm{o}}$ & Título dos trabalhos & Ano & Métodos & Aspectos relevantes & Autores & $\begin{array}{l}\text { Bases de } \\
\text { dados }\end{array}$ \\
\hline 01 & $\begin{array}{l}\text { Equipe de } \\
\text { enfermagem: } \\
\text { conhecimento acerca } \\
\text { do manuseio de } \\
\text { drogas antineoplásicas } \\
\end{array}$ & \multirow{5}{*}{2011} & $\begin{array}{l}\text { Descritivo } \\
\text { exploratório } \\
\text { com abordagem } \\
\text { quantitativa }\end{array}$ & $\begin{array}{l}\text { Acidente ocupacional } \\
\text { relacionado ao } \\
\text { antineoplásico }\end{array}$ & $\begin{array}{l}\text { D., SJH; M, } \\
\text { KF; O PJM; } \\
\text { M, AH; M, } \\
\text { LHM }\end{array}$ & LILACS \\
\hline 02 & $\begin{array}{l}\text { Fatores de risco para } \\
\text { câncer cervical em } \\
\text { mulheres assistidas } \\
\text { por uma equipe de } \\
\text { saúde da família em } \\
\text { Cuiabá, MT, Brasil } \\
\end{array}$ & & $\begin{array}{l}\text { Quantitativa e } \\
\text { Descritiva }\end{array}$ & $\begin{array}{l}\text { Agentes de saúde como } \\
\text { facilitadoras e rastreadoras } \\
\text { das pendências de exames } \\
\text { para mulheres }(C P)\end{array}$ & $\begin{array}{l}\text { D, SJH; M, } \\
\text { KF; O, PJM; } \\
\text { M, AH; M, } \\
\text { LHM }\end{array}$ & LILACS \\
\hline 03 & $\begin{array}{l}\text { Espiritualidade na } \\
\text { equipe interdisciplinar } \\
\text { que atua em cuidados } \\
\text { paliativos às pessoas } \\
\text { com câncer }\end{array}$ & & $\begin{array}{l}\text { Descritiva e } \\
\text { exploratória } \\
\text { qualitativa }\end{array}$ & $\begin{array}{l}\text { Visão holística , a } \\
\text { espiritualidade como } \\
\text { dimensão }\end{array}$ & $\begin{array}{l}\text { A, ICO; T, } \\
\text { MB; P, AR; } \\
\text { P, JS }\end{array}$ & LILACS \\
\hline 04 & $\begin{array}{l}\text { Qualidade de vida de } \\
\text { adolescentes curados } \\
\text { de câncer - uma } \\
\text { abordagem qualitativa } \\
\text { e interdisciplinar }\end{array}$ & & $\begin{array}{l}\text { Exploratório } \\
\text { descritivo com } \\
\text { abordagem } \\
\text { qualitativa } \\
\text { interdisciplinar }\end{array}$ & $\begin{array}{l}\text { Após a cura, a volta a } \\
\text { sociedade para crianças e } \\
\text { adolescentes }\end{array}$ & V, VD; S, KV & LILACS \\
\hline 05 & $\begin{array}{l}\text { Fatores associados } \\
\text { ao linfedema em } \\
\text { pacientes com câncer } \\
\text { de mama }\end{array}$ & & $\begin{array}{l}\text { Corte } \\
\text { transversal }\end{array}$ & $\begin{array}{l}\text { Linfedema prevenção, } \\
\text { enfoque no fisioterapeuta }\end{array}$ & $\begin{array}{l}\text { P, DMF; L, } \\
\text { ICG; R VO; } \\
\text { C, MG }\end{array}$ & SciELO \\
\hline 06 & $\begin{array}{l}\text { Tratamento paliativo: } \\
\text { perspectiva da família }\end{array}$ & \multirow{3}{*}{2012} & $\begin{array}{l}\text { Fenomenologia } \\
\text { sociologia de } \\
\text { Alfred Schutz }\end{array}$ & $\begin{array}{l}\text { Família como elemento } \\
\text { do cuidado, demanda } \\
\text { espiritual, abordagem } \\
\text { paliativa inter }\end{array}$ & $\begin{array}{l}\text { N, MGS; R, } \\
\text { BMRD }\end{array}$ & LILACS \\
\hline 07 & $\begin{array}{l}\text { A inserção } \\
\text { do psicólogo } \\
\text { na residência } \\
\text { multiprofissional } \\
\text { em saúde: um relato } \\
\text { de experiência em } \\
\text { oncologia }\end{array}$ & & $\begin{array}{l}\text { Relato de } \\
\text { experiência }\end{array}$ & $\begin{array}{l}\text { Residência multidisciplinar } \\
\text { em oncologia e sua } \\
\text { importância }\end{array}$ & $\begin{array}{l}\text { M, JL;C, } \\
\text { ESA; S, AM }\end{array}$ & LILACS \\
\hline 08 & $\begin{array}{l}\text { Fatores preditores } \\
\text { da esperança entre } \\
\text { mulheres com } \\
\text { câncer de mama } \\
\text { durante o tratamento } \\
\text { quimioterápico }\end{array}$ & & $\begin{array}{l}\text { Prospectivo } \\
\text { de corte } \\
\text { longitudinal }\end{array}$ & $\begin{array}{l}\text { O transtorno causado } \\
\text { pela dor necessita de uma } \\
\text { assistência interdisciplinar, } \\
\text { além da avaliação, terapia } \\
\text { farmacológica, e não } \\
\text { farmacológica o transtorno } \\
\text { causado pela dor precisa } \\
\text { assistência interdisciplinar }\end{array}$ & $\begin{array}{l}\text { B, ACS; G, } \\
\text { SAA }\end{array}$ & SciELO \\
\hline
\end{tabular}




\begin{tabular}{|c|c|c|c|c|c|c|}
\hline $\mathrm{N}^{\mathrm{o}}$ & Título dos trabalhos & Ano & Métodos & Aspectos relevantes & Autores & $\begin{array}{l}\text { Bases de } \\
\text { dados }\end{array}$ \\
\hline 09 & $\begin{array}{l}\text { Prevalência de } \\
\text { Neoplasias, Cárie } \\
\text { e Gengivite em } \\
\text { Pacientes Oncológicos } \\
\text { Pediátricos no } \\
\text { Município de Belém. } \\
\text { Pará }\end{array}$ & \multirow{6}{*}{2013} & Grupo controle & $\begin{array}{l}\text { Implantação de gabinetes } \\
\text { odontológicos durante } \\
\text { a terapia oncológica. } \\
\text { Cuidado ANTES E DEPOIS } \\
\text { dos antineoplásicos } \\
\text { principalmente crianças }\end{array}$ & $\begin{array}{l}\text { F PBA; N, } \\
\text { AJS }\end{array}$ & LILACS \\
\hline 10 & $\begin{array}{l}\text { O adoecer e } \\
\text { sobreviver ao } \\
\text { câncer de mama: a } \\
\text { vivência da mulher } \\
\text { mastectomizada }\end{array}$ & & Qualitativo & $\begin{array}{l}\text { Avaliação da mulher } \\
\text { mastectomizada, } \\
\text { promover a articulação } \\
\text { dos cuidados com a equipe } \\
\text { multi alimentação, fisio, } \\
\text { atividade física, abordagem } \\
\text { multidimensional e inter, } \\
\text { articulação com demais } \\
\text { áreas }\end{array}$ & $\begin{array}{l}\text { P, CM; P, } \\
\text { BK; M, RM; } \\
\text { C, DH; W, } \\
\text { WP }\end{array}$ & LILACS \\
\hline 11 & $\begin{array}{l}\text { Processo clínico e } \\
\text { integridade do cuidar } \\
\text { em enfermagem de } \\
\text { pessoas com câncer: } \\
\text { Investigação piloto } \\
\end{array}$ & & $\begin{array}{l}\text { Método } \\
\text { descritivo e } \\
\text { técnica de } \\
\text { autorrelato }\end{array}$ & $\begin{array}{l}\text { Integralidade, saude } \\
\text { holística, conexão espiritual, }\end{array}$ & $\begin{array}{l}\text { S, I; F, LR; C, } \\
\text { AC; K, CCF; } \\
\text { S, AV }\end{array}$ & LILACS \\
\hline 12 & $\begin{array}{l}\text { Dificuldades da } \\
\text { enfermagem na } \\
\text { utilização do lúdico } \\
\text { no cuidado à } \\
\text { criança com câncer } \\
\text { hospitalizada }\end{array}$ & & $\begin{array}{l}\text { Descritiva com } \\
\text { abordagem } \\
\text { qualitativa }\end{array}$ & $\begin{array}{l}\text { O lúdico como prática a } \\
\text { criança hospitalizada com } \\
\text { câncer }\end{array}$ & $\begin{array}{l}\text { Di, JRB; S, } \\
\text { LF; M, ACM; } \\
\text { S, RS }\end{array}$ & LILACS \\
\hline 13 & $\begin{array}{l}\text { Atuação do } \\
\text { enfermeiro em } \\
\text { oncologia na } \\
\text { perspectiva da } \\
\text { genética e genômica }\end{array}$ & & Reflexão & $\begin{array}{l}\text { Equipe multiprofissional } \\
\text { e interdisciplinar de } \\
\text { aconselhamento genético } \\
\text { oncológico; enfermeiros } \\
\text { geneticistas; a maioria } \\
\text { dos enfermeiros nao teve } \\
\text { iniciativa de confrontar-se } \\
\text { com avanços; déficit de } \\
\text { conhecimento falta de fontes } \\
\text { de informações atualizadas } \\
\text { e contextualizadas a } \\
\text { sua prática, central de } \\
\text { planejamento e execução } \\
\text { de cuidado baseado em } \\
\text { genômica }\end{array}$ & & SciELO \\
\hline 14 & $\begin{array}{l}\text { Morte digna da } \\
\text { criança: percepção de } \\
\text { enfermeiros de uma } \\
\text { unidade de oncologia }\end{array}$ & & $\begin{array}{l}\text { Exploratório } \\
\text { descritivo com } \\
\text { abordagem } \\
\text { qualitativa } \\
\end{array}$ & $\begin{array}{l}\text { Cuidado holístico, cuidado } \\
\text { humanizado, cuidados } \\
\text { paliativos= criança trabalho } \\
\text { em equipe morte digna }\end{array}$ & $\begin{array}{l}\text { S, LF; M, } \\
\text { MD; S, L; P, } \\
\text { K; S, MR; } \\
\text { B, RS }\end{array}$ & SciELO \\
\hline
\end{tabular}




\begin{tabular}{|c|c|c|c|c|c|c|}
\hline $\mathrm{N}^{\mathrm{o}}$ & Título dos trabalhos & Ano & Métodos & Aspectos relevantes & Autores & $\begin{array}{l}\text { Bases de } \\
\text { dados }\end{array}$ \\
\hline 15 & $\begin{array}{l}\text { Visão dos } \\
\text { profissionais sobre } \\
\text { o seu tratamento } \\
\text { no programa de } \\
\text { internação domiciliar } \\
\text { interdisciplinar } \\
\text { oncológico: uma } \\
\text { realidade brasileira }\end{array}$ & 2014 & $\begin{array}{l}\text { Descritivo com } \\
\text { abordagem } \\
\text { qualitativa }\end{array}$ & $\begin{array}{l}\text { A interdisciplinaridade } \\
\text { é entendida como uma } \\
\text { necessidade intrínseca } \\
\text { para referenciar as } \\
\text { práticas, integração de } \\
\text { saberes, diversidade } \\
\text { de olhares CUIDADO } \\
\text { PALIATIVO, inter } \\
\text { relações de competências } \\
\text { profissionais; a necessidade } \\
\text { de prática interdisciplinar } \\
\text { proporciona a troca de } \\
\text { informações; a equipe } \\
\text { mantém forte vínculo;a } \\
\text { equipe se auto ajuda, } \\
\text { necessita preparo para } \\
\text { atuação interdisciplinar( } \\
\text { bases de formação } \\
\text { curricular); preconiza o } \\
\text { cuidado integral;favorece a } \\
\text { produção científica }\end{array}$ & $\begin{array}{l}\text { P, AR; T, } \\
\text { MB; P, DD; } \\
\text { Ay, SC; A, I; } \\
\text { J, LR }\end{array}$ & SciELO \\
\hline 16 & $\begin{array}{l}\text { Trabalho emocional } \\
\text { e gestão de emoções } \\
\text { em equipes de saúde } \\
\text { oncológicas: um } \\
\text { estudo qualitativo }\end{array}$ & & $\begin{array}{l}\text { Qualitativo } \\
\text { com abordagem } \\
\text { fenomenológica }\end{array}$ & Comunicação interpessoal & $\begin{array}{l}\text { C, CMS; B, } \\
\text { E; P, PMSD; } \\
\text { O, DC }\end{array}$ & LILACS \\
\hline 17 & $\begin{array}{l}\text { Cuidados paliativos } \\
\text { em oncologia } \\
\text { pediátrica:percepções, } \\
\text { saberes e práticas na } \\
\text { perspectiva da equipe } \\
\text { multiprofissional }\end{array}$ & 2015 & $\begin{array}{l}\text { Qualitativa } \\
\text { do tipo } \\
\text { exploratório } \\
\text { descritivo }\end{array}$ & $\begin{array}{l}\text { VISÃO ampliada dos } \\
\text { sujeitos, reflexão contínua } \\
\text { discutido em equipe, } \\
\text { a equipe possui visão } \\
\text { ampliada dos sujeitos; } \\
\text { discussão em equipe; } \\
\text { construção coletiva; } \\
\text { comunicação; grupo que } \\
\text { trabalha junto há mais } \\
\text { tempo }\end{array}$ & $\begin{array}{l}\text { S, AF; I, HB; } \\
\text { M, MGC; B, } \\
\text { DZA }\end{array}$ & SciELO \\
\hline 18 & $\begin{array}{l}\text { Os cuidados } \\
\text { paliativos como } \\
\text { política pública: notas } \\
\text { introdutórias }\end{array}$ & 2016 & Introdutório & $\begin{array}{l}\text { Integração de diferentes } \\
\text { áreas de conhecimento, } \\
\text { atenção domiciliar com } \\
\text { ações intersetoriais } \\
\text { cuidados planejados } \\
\text { ações integradas, ANCP, } \\
\text { introdução para REDE, } \\
\text { modelos de atenção à saúde }\end{array}$ & $\mathrm{F}, \mathrm{A}$ & SciELO \\
\hline
\end{tabular}

Dentre os artigos incluídos na revisão integrativa, 12 são de autoria de enfermeiros, 4 têm entre seus autores odontólogos e psicólogos, 2 foram redigidos por médicos e assistentes sociais.

No momento não foi encontrado uma justificativa baseada em estudos anteriores que fundamentam o número de Enfermeiros pesquisadores.

Analisando os profissionais que mais investigam o assunto da interdisciplinaridade, foi constatado que $66,6 \%$ foram Enfermeiros graduados em fase de mestrado e doutorado, 22,2\% profissionais odontólogos e psicólogos em fase de especialização, e por fim 11,1 \% médicos e assistentes sociais em fase de especialização.

A prática, baseada em evidências, movimento estruturado como facilitador da tomada de decisão, pode ser definida como um processo de busca, 
avaliação e aplicação de evidências científicas para o tratamento e gerenciamento da saúde (HAMMER, 1999). É uma abordagem que possibilita a melhoria da qualidade da assistência prestada ao paciente e incentiva o profissional de saúde buscar conhecimento científico por meio do desenvolvimento de pesquisas ou aplicação na sua prática dos resultados encontrados na literatura (GALVÃO, 2002)

Em relação ao tipo de periódicos nos quais foram publicados os artigos incluídos na revisão, 14 foram publicados em Revistas de Enfermagem, 1 em Revista de Odontopediatria, 1 Revista de Psicologia, 1 em Caderno de Saúde Coletiva e 1 em Revista de Ginecologia e Obstetrícia.

Avaliando a proposta inicial desta revisão em relação ao objetivo do estudo, observa-se que a questão interdisciplinar está sendo abordada por profissionais de diferentes áreas, no intuito de melhorar o atendimento do paciente oncológico, já que a manutenção da vida seja paliativa ou curativa está acontecendo no próprio domicílio e não mais restrito a instituições de saúde.

Duarte e Diogo (2000) apontam: a transição demográfica, a mudança no perfil epidemiológico da população, no qual se evidencia um aumento das doenças crônico-não transmissíveis; os custos do sistema hospitalar cada vez mais elevado; o desenvolvimento de equipamentos tecnológicos, que têm possibilitado maior taxa de sobrevida das pessoas; o aumento da procura por cuidados de saúde; o interesse dos profissionais de saúde por novas áreas de atuação; a exigência por maior privacidade, individualização e humanização da assistência à saúde, além da necessidade de maior integração da equipe profissional com o cliente e sua família. Existindo a modalidade de atenção domiciliar.

No entanto, o vínculo com o grupo multiprofissional não está determinado de forma interdisciplinar, como observou-se na pesquisa. Cada área está se organizando individualmente para melhorar a qualidade de vida dos pacientes, não existe um eixo norteador, o serviço acontece de maneira aleatória conforme demanda e necessidade.

O processo de trabalho interdisciplinar encontra algumas dificuldades visto que, segundo SILVA e HAHN(2012) os profissionais compreendem suas atribuições na equipe e sentem reconhecimento de seu trabalho pelos colegas. Entretanto, estresse e diferenças de personalidade, a sobreposição de papéis, injustiças no trabalho, dificuldade em compreender a linguagem utilizada pelos colegas e grande demanda de trabalho conferem em aspectos negativos. Sendo de suma importância a integração entre os membros da equipe para que esta avalie seu processo de trabalho de modo a resultar em melhorias no trabalho e na assistência ao paciente.

No estudos avaliado, foi observado a presença de um grupo interdisciplinar atuante em atendimento hospitalar, quando paciente encontrase em cuidados paliativos em fase final da doença. 
Quando se compartilha cotidianamente situações inerentes à facticidade existencial do viver em cuidados paliativos e sua família, aflora na equipe, compreensões peculiares ao momento vivido. Tais compreensões promovem o repensar sobre o cuidado em suas múltiplas dimensões e vêm ressignificar o foco de atenção nas relações com os pacientes e familiares e entre os profissionais da própria equipe. explicita fatores que podem vir a qualificar tais práticas e revelar a importância de poder refletir e discutir mais sobre cuidados paliativos com toda a equipe multidisciplinar. (SILVA A.F. et al, 2015)

Igualmente visto em um programa de internação domiciliar existente em uma única cidade do estado do Rio Grande do Sul.

A necessidade da prática interdisciplinar, como estratégia no campo da saúde, justifica-se pela potencialidade da mesma em proporcionar a troca de informações e de críticas entre os profissionais da saúde e das humanas, ampliar a formação geral dos especialistas e questionar a possível acomodação dos profissionais com a assistência oferecida. Alguns profissionais referem buscar subsídios em cursos de pós-graduação para constituir de fato, a prática interdisciplinar da equipe.( PORTO et. al., 2014)

Por se tratar de uma abordagem inovadora, haja vista que faltam leis e normatizações para implantação desse recurso em outros lugares, torna-se necessária a integração de diferentes áreas de conhecimento para desvendar problemas de natureza pública e política.

Outros estudos chamaram a atenção para a saúde holística e espiritualidade, pouco estudado atualmente pelos profissionais da saúde: Para ARRIEIRA et.al.(2011) a espiritualidade é uma via de mão dupla, na qual ocorrem trocas solidárias e afetivas entre profissionais e pacientes, possibilitando crer num ser transcendente não necessariamente ligado às religiões. Em outras palavras, significa a conexão do paciente com os processos inconscientes do existir na busca de sentido à vida, além de atuar como harmonizador no processo de trabalho da equipe interdisciplinar.

\section{CONCLUSÃO}

No âmbito da saúde, sugere-se mais aprofundamento das políticas públicas bem como, estratégias que visam o trabalho de forma interdisciplinar, tornando o atendimento do paciente oncológico mais completo, de qualidade e humanizado. De fato, o tema interdisciplinaridade ainda é pouco abordado, levando em consideração as questões de integralidade do cuidado e a possibilidade de mudança do modelo técnico assistencial da atualidade.

Essas normatizações poderiam ser semelhantes ao processo de acreditação hospitalar que busca um atendimento de qualidade, seguro e integral mediante normas e rotinas. Neste processo, a integralidade é incentivada e buscada. 
Faz-se necessário mais estudos que contribuam para a formação de profissionais com um olhar interdisciplinar. Desta forma, as relações de trabalho ocorrerão de forma mais horizontal, garantindo a qualidade e segurança da assistência.

Foi possível demonstrar a ampla variedade de profissionais que são importantes para o tratamento do paciente para que o mesmo seja atendido de forma integral, como é preconizado. O tema é complexo e exige mais reflexões e discussões acerca do assunto, envolve demandas públicas e produção de normas e diretrizes para a execução dessa forma de trabalho.

O Presente estudo contribui para o debate em torno dos cuidados multiprofissionais ao paciente oncológico de forma interdisciplinar nas diferentes abordagens de tratamento, seja curativo, adjuvante, neoadjuvante ou paliativo.

A revisão também possibilitou observar que a literatura atual diminuiu as publicações relacionadas ao trabalho interdisciplinar na segunda metade do período estudado.

A busca de conhecimento e atualização precisa ser constante para qualquer profissional, através de leitura, reflexões, discussões. Sendo assim, esse estudo é apenas uma ferramenta para instigar ações inovadoras que ampliem a troca de saberes.

\section{REFERÊNCIAS}

ARRIEIRA I.C.O, et. al., Espiritualidade na equipe interdisciplinar que atua em cuidados paliativos às pessoas com câncer. Rev. Cuid. Saude 2011 Abr/jun; 10(2):314321.

BRASIL, Ministério da Saúde: - Clínica Ampliada, Equipe de Referência e Projeto Terapêutico Singular, Secretaria de Atenção à Saúde, Núcleo Técnico da Política Nacional de Humanização - 2.ed..- Brasília, 2007. Ministério da Saúde.

DUARTE, Y. A. O.; DIOGO, M. J. E. Atendimento domiciliar : um enfoque gerontológico. São Paulo: Atheneu,2000.

GALVÃO CM, Sawada NO, Rossi LA. A prática baseada em evidências: considerações teóricas para sua implementação na enfermagem perioperatória. Rev Latino-am Enfermagem 2002 setembro-outubro; 10(5):690-5.

GANONG LH. Integrative reviews of nursing research. Res Nurs Health. 1987; Mar 10(1):1-11

GONZÁLEZ, A. D. ALMEIDA, M. J. Integralidade da saúde - norteando mudanças na graduação dos novos profissionais.Ciência e Saúde Coletiva,.15(3):757-762, 2010. 
HAMMER S. Evidence-based practice. In: Hamer S, Collinson G. Achieving evidencebased practice: a handbook for practitioners. London: Baillière Tindall; 1999. p. 3-12

INSTITUTO NACIONAL DO CÂNCER. Estimativas da incidência e mortalidade por câncer no Brasil. Rio de Janeiro: INCA; 2002

ROMAN AR, Friedlander MR. Revisão integrativa de pesquisa aplicada à enfermagem. Cogitare Enferm. 1998 Jul-Dez; 3(2):109-12.

PORTO A.R Visão dos profissionais sobre seu trabalho no programa de internação domiciliar interdisciplinar oncológico: uma realidade brasileira. av.enferm., XXXII (1): 72-79, 2014

SILVA D. S.,HAHN G.Processo de trabalho em oncologia e a equipe multidisciplinar.Caderno pedagógico, Lajeado, v. 9, n. 2, p. 125-137, 2012125

SILVA A. F. et al. Cuidados paliativos em oncologia pediátrica: percepções, saberes e práticas na perspectiva da equipe multiprofissional. Rev Gaúcha Enferm. 2015 jun;36(2):56-62.

WHO. National câncer control programmes: policies and managerial guidelines. 2nd. Edition, Geneve: OMS, 2002. 